\title{
Substitution: right or wrong?
}

\author{
J. C. BARFIELD \\ F.P.S.
}

South East Metropolitan Regional Hospital Board

\begin{abstract}
Summary
Drug economy has always been and will continue to be important for hospitals. Substitution, as an aid to economy, requires reappraisal in the light of our developing knowledge of formulation and resultant bio-availability. The continued unqualified use of generic names in teaching and prescribing requires reexamination as a part of the problem and this highlights the need for better communication between medicine and pharmacy.
\end{abstract}

IT was not long after accepting the invitation to speak that I realized that I did not know the overall black and white answer to the question, but I have taken consolation in assuming that $I$ am not alone. I will briefly propose a definition for substitution, trace the changing relationship between doctors and pharmacists, indicating where and why substitution occurs and how all this now looks in the light of our changing knowledge about drugs.

In relation to drug prescribing, substitution means the supply against prescription of an alternative drug form or formulation, an alternative brand of drug for a given brand or the supply of a generic, or unbranded drug, for a branded drug. The last example, generic for branded, illustrates the most commonly occurring form of substitution and is the one I will use unless the term is qualified further. Some drugs are only available in generic form and some proprietary brands are very old, for example the Luminal brand of phenobarbitone, so that the use of the generic drug in such circumstances is not usually thought of as substitution, i.e. the substitution of one drug entity for another, for example Erythromycin tablets for Penicillin V tablets, is rarely undertaken by pharmacists except in cases of extreme emergency where the provision of some treatment is the most important consideration, e.g. the substitution of one simple analgesic for another at a weekend.

Traditionally, a doctor's prescription is an order and not a request to the pharmacist who dispenses or supervises the dispensing of the drug concerned. This Architect and Artisan relationship held well into the 1940s, when, although chemotherapy was established and other synthetic drugs were appear- ing, the older physician retained a knowledge of Materia Medica and simple compounding. Today the medical student does not have time to absorb all the complexities of modern drugs in spite of the efforts of his Professor of Clinical Pharmacology and indeed he will end up with only a superficial knowledge of prescribing as such. Later he will work with Hospital Consultants with a specialized knowledge of a field of medicine and the drugs appropriate to that field. In general practice and in many aspects of hospital work he will find a formidable range of therapeutic agents to choose from, both branded and generic.

Pharmacy has also changed. The traditional training emphasis on the processing of galenicals and the subtle skills and arts of the dispensing counter, guided by pharmacopoeial standards based principally on chemical analytical methods, has been replaced by a broadly based scientific degree which includes pharmacology and biopharmaceutics. The manipulative aspects of dispensing and distribution are slowly being delegated to pharmacy technicians. At the same time the development and standardization of manufacture of drug products have passed and are passing steadily to the pharmaceutical industry. The new generation pharmacist can no longer support the physician solely through bench dispensing skills. His knowledge requires to be harnessed to help in the clinical team but too often the organization of hospital and general practice do not readily provide for this.

In this changing environment the sustained reason for substitution has been financial economy. Up to the mid-1940s hospital pharmacopoeias and local and national formularies contained many cheap and simple drug formulations as alternatives to proprietary forms and the local authority and voluntary hospitals were operating on very limited funds. The war years taught us to manage without alcohol, glycerin and vegetable drugs from overseas sources. Chemical purity seemed a sure enough guarantee of activity and the pressing need for economy justified collective substitution. It seemed logical and right. The cynical may doubt the value of remedies but this view may be counterbalanced, in relation to 
substitution, by the fact that the control standards applied to proprietary and generic drugs were of a similar order.

Since the outset in 1948, costs for the National Health Service have exceeded estimates and drug costs and drug companies have received no little amount of attention. Although the service is based on the freedom of the clinician to prescribe what he considers best for his patient the Department of Health and Social Security has for some time regularly circularized bar charts comparing the cost of selected branded drugs with their generic counterparts. The prescriber is thus made aware of relative costs. Although a footnote disclaims that the examples on any one chart have comparable therapeutic effect, the implication is that if the prescriber selects, or substitutes, one of the cheaper alternatives money is saved without serious detriment to the patient. Medical Schools, authors of research papers and hospital pharmacists have promoted the use of approved names as standard practice to simplify communication together with the use of the British National Formulary. In hospital, in addition to agreements with individual prescribers, pharmacists have frequently sought and obtained the authority to substitute BP and BPC drugs for their branded counterparts and have been encouraged in this approach by regional and national contracts for unbranded drugs. It is also common hospital practice to prepare equivalents of branded topical and oral preparations. Often hospital developed formulations have led to the introduction of a BP or BPC formulation. In general practice substitution is not permitted under the terms of contract with Executive Councils, whose medical advisers, nevertheless, exert pressure on general practitioners considered to be 'excessive prescribers'. Certainly in the 1950 s and 1960s the pharmaceutical industry did not help itself much in relation to substitution. There have been examples of molecular roulette, duplicate marketing, 'me too' products and the marketing of compounds of a chemical series with little clear advantage over their predecessors. Apart from the need to economize, the use of generic drugs has offered an attractive rationalization from the excessive number of products available. Too often propaganda for branded products has been slanted and exaggerated.

The advance of pharmaceutical technology may owe something to that unknown nurse who observed that Blauds Pills administered to patients were rattling in the bed pans. The importance of disintegration rates of tablets and the ensuing dissolution rates of drugs, the $\mathrm{pH}$ and composition of body fluids, whether the drug is present as its base, salt or ester and the resultant effect on absorption rates has been known for some time. We have realized the effect of isomers and particle size in both injections and oral dose forms. More recently the importance of bio-availability, genetic variations in patients, excretion pathways and drug interactions and toxicity mechanisms have been recognized. The placebo effect and the intricacies of statistical interpretation of clinical trials have indicated the difficulty of accurately assessing the worth of a drug. These determinations require time, often expensive equipment and the co-ordinating work of diverse professional skills.

Today only a large organization can afford the total effort that is required. The small pharmaceutical company devoted to processing generic drugs is unlikely to be able to meet this emerging requirement. Sir Derrick Dunlop wrote earlier this year: 'I regret my passionate insistence that only generic drugs must be prescribed thereby effecting great economies with no detriment to patients. The generic names refer only to the active chemical agent rather than the product given to the patient. That the problem of bio-availability is an important one has been emphasized recently in regard to digoxin and it is obviously dangerous to assume, as I used to do, that generically equivalent products are invariably of equal therapeutic potency to branded products'. I can remember one large pharmaceutical company which had the embarrassment of withdrawing a calcium salt as an excipient from liquid tetracycline syrup and $I$ understand variation in digoxin availability from tablets were traced to a revised tablet granulation process by another reputable manufacturer. The important point is not that reputable companies had these difficulties with branded products but that they were recognized, detected and corrected.

The object of substitution is economy. I have often wondered just what the apparent and real savings are in relation to the use of substitutes. Perhaps we have been too preoccupied with savings at source rather than selection and use. Nevertheless, the cost of drugs is very substantial and a high proportion of the total level of expenditure can be traced to less than a hundred particularly expensive items, whether this analysis is made for hospitals or general practice. Our under-managed and over-administered hospital service does not relate directly the cost of a drug to the effective treatment of patients or the effective use of hospital resources. Thus, for example, drug costs are totalled under one head, and length of stay under another. The precise assessment of the effect of drugs is, therefore, obscured.

\section{Conclusions}

(1) Substitution was right in hospitals up to 10 years ago but only in the light of knowledge then available.

(2) Hospitals provide a non-profit making social 
service and predictably will never have enough money to fulfil all desired aims. Drug economy will therefore continue to be important. Substitution is not the only approach.

(3) As an aid to economy, although drug substitution is an apparently simple way of reducing costs, its application requires more searching appraisal. Drugs offered for regional contracts required detached investigation.

(4) The greater benefit to patients and probably the best single saving in real terms is to be achieved by using the most efficient product of an available drug judged by modern chemical, physical and human biological tests whether this be branded or generic in origin.

(5) Today blind substitution is wrong. The traditional roles of physician and pharmacist need reexamination. Is it still true that most clinicians have enough information and detailed knowledge to insist that a certain brand of a drug should be used? Similarly, does the pharmacist have all the information needed to assess a substitute? A collective approach is required and access to authoritative information, and hospitals might consider the value of a multidisciplinary drug evaluation team.

(6) Greater co-operation is required between medicine and pharmacy in drug prescribing, selection and monitoring. Current developments in the hospital pharmaceutical service, particularly in drug information, ward pharmacy and quality control, indicate that the need for change has some impetus. In my view, these developments do not clash with the development of clinical pharmacology as a medical speciality or the activities of biochemists.

(7) Substitition in general practice pharmacy is wrong because of the nature of the contractural services. However, some discretion to supply alternative brands would reduce inconvenience to patients in some instances.

(8) The propaganda provided by the Department of Health and Social Security gives the impression that drug costs are independent of patients and the incidence of disease and encourages substitution.

\section{References}

Dunlop, SIR D. (1973) Confession is good for the soul. World Medicine, 8, No. 12, 35. 